Journal of Social Sciences (COES\&RJ-JSS)

ISSN (E): 2305-9249 ISSN (P): 2305-9494

Publisher: Centre of Excellence for Scientific \& Research Journalism, COES\&RJ LLC

Online Publication Date: $1^{\text {st }}$ July 2017

Online Issue: Volume 6, Number 3, July 2017

https://doi.org/10.25255/jss.2017.6.3.618.622

\title{
Product of conjugates
}

\author{
Ms. Normalah P. Solaiman \\ Department of Education \\ Autonomous Region in Muslim Mindanao, Philippines \\ Analyn P. Guro \\ Mindanao State University \\ Main Campus, Marawi City, Philippines
}

\begin{abstract}
:
This qualitative study is an extension of the previous research of Solaiman (2016) in which the standard form of a factorable polynomial of nth terms is introduced. Its aim is to investigate other forms of factorable polynomials. It is $\mathrm{x}^{\mathrm{n}}-\mathrm{y}^{\mathrm{n}}$ that gives the initial form of the product of the first known binomial conjugates. Another form is the trinomial $x^{4 n}-$ $(x y)^{2 n}+y^{4 n}$ which is a product of $x^{2 n}-(x y)^{n}+y^{2 n}$ and $x^{2 n}+(x y)^{n}+y^{2 n}$ for some positive integer $n$. The result leads to a form of the product of two conjugate polynomials with $\mathrm{n}$ terms where $\mathrm{n}$ is a positive integer. This paper illustrated that there are still many polynomials which are undiscovered as factorable. Thus, it motivates that students should explore mathematical expressions and formulate equations to develop their mathematical thinking skills to become critical thinkers in the future.
\end{abstract}

Keywords :

Factorable polynomials, binomial conjugates

\section{Citation:}

Solaiman, Ms. Normalah P.; Guro, Analyn P. (2017) ; Product of conjugates; Journal of Social Sciences (COES\&RJ-JSS), Vol.6, No.3, pp:618-622; https://doi.org/10.25255/jss.2017.6.3.618.622. 


\section{Introduction}

Inventions of technology are applications of the mathematical equations or algebraic expressions. Polynomials are algebraic expressions that have some applications in the form of areas and perimeters of geometric shapes and even businesses. This is the power of the number sense.

Exploring polynomials to discover many concepts gives hints of how the existing theorems, postulates, or any mathematical concepts were derived and discovered. This made the possibility of adding new ideas; for instance, the rational zeros of $\mathrm{x}^{\mathrm{n}}-\mathrm{y}^{\mathrm{n}}$ shows that the expression, $x^{2}-y^{2}$, is indeed factorable. The product of $(a x)^{2 n}-(a x b y)^{n}+(b y)^{2 n}$ and $(a x)^{2 n}+(a x b y)^{n}+(b y)^{2 n}$ is another elaboration of two conjugates whose product is a factorable polynomial. This study illustrated many products of conjugates and even formulated a polynomial of $\mathrm{n}$ terms, which is a product of conjugates, to add concepts to the existing types of factoring introduced in many mathematics textbooks.

\section{Statement of the Problem}

1. What are the factors of $x^{4 n}+(x y)^{2 n}+y^{4 n}$ ?

2. What are the factors of $x^{8 n}+(x y)^{6 n}+(x y)^{4 n}+(x y)^{2 n}+\left(y^{8 n}\right)$ ?

3. What are the factors of $x^{4 n}+(x y)^{4 n-2 d}+(x y)^{4 n-4 d}+\ldots+(x y)^{2 d}+y^{4 n}$ ?

\section{Theoretical Framework}

Some of the theories that are utilized in this study are exploratory, transitive properties of equality, symmetric properties of equality, and rational zeros of polynomials.

Exploratory theory of learning explains that learning is achieved through exploring concepts. Transitive property of equality describes that if for some real numbers $a, b$, and $c$ and if $a$ is equal to $b$ and $b$ is equal to $c$ then $a$ is equal to $c$. Symmetric property of equality illustrates that for some real numbers $\mathrm{x}$ and $\mathrm{y}$, if $\mathrm{x}$ is equal to $\mathrm{y}$ then $\mathrm{y}$ is equal to $\mathrm{x}$. Rational zeros of a polynomial elaborates that a real number a is a root of a polynomial if it is satisfy the equation after substituting it.

\section{Conceptual Framework}

This study was influenced by the discovery of Solaiman (2016) of the expression, $\mathrm{x}^{4}-\mathrm{x}^{2}+$ 1 , which is factorable into $x^{4}-x^{2}+1$ and $x^{4}+x^{2}+1$. This started from factoring $x^{6-} y^{6}$ using difference of two perfect squares and difference of two perfect cubes. It is observed from the difference of two perfect squares, difference of two perfect cubes, and the trinomial conjugates that product of conjugates are primarily found in the polynomials having prime number of terms and whose powers of its terms are sequence. Because of the eagerness of finding more concepts of the possibility of a polynomial with 5 , 7 , or more than 7 terms as a product of two conjugates, a polynomial form of $\mathrm{n}$ terms which can be a product of two conjugates was established in this study.

\section{Methodology}

This study is a qualitative research that investigated the polynomials which can be found as product of two conjugates. The researcher (Solaiman, 2016) observed that this case can be possible only if the number of terms in a polynomial is odd leaving some of its terms eliminated after multiplying. She also found out that the powers of the terms in a polynomial should be sequential. That is, powers have a constant difference. 


\section{Product of conjugates}

Using properties of real numbers and laws of equality, a form of a factorable polynomial with odd number of terms and which is a product of two conjugates is derived.

\section{Presentation of Results}

The first problem in this study is to show the factors of $x^{4 n}+(x y)^{2 n}+y^{4 n}$. It is called as lemma 1 as follows.

Lemma 1. Trinomial of the form $x^{4 n}+(x y)^{2 n}+y^{4 n}$

Let $n$ be a positive integer then $x^{4 n}+x^{2 n}+1$ can be factored as $\left(x^{2 n}+x^{n}+1\right)\left(x^{2 n}-x^{n}+1\right)$. Proof: Adding the left side of the expression with $\left(x^{3 n}+x^{2 n}+x^{n}\right)-\left(x^{3 n}-x^{2 n}+x^{n}\right)$ and using factoring by grouping,

$$
\left(x^{4 n}+x^{2 n}+1\right)+\left(x^{3 n}+x^{2 n}+x^{n}\right)-\left(x^{3 n}-x^{2 n}+x^{n}\right)=\left(x^{2 n}+x^{n}+1\right)\left(x^{2 n}-x^{n}+1\right) .
$$

Similarly, multiplying the right side factors gives the left side expression as product. Example. Find the factors of

$$
\text { 1. } x^{8}+x^{4}+1
$$

2. $m^{24}+m^{12}+1$

Solution:

$$
\begin{aligned}
\text { 1. } \mathrm{x}^{8}+\mathrm{x}^{4}+1= & \left(\mathrm{x}^{4}+\mathrm{x}^{2}+1\right)\left(\mathrm{x}^{4}-\mathrm{x}^{2}+1\right) \\
= & \left(\mathrm{x}^{2}-\mathrm{x}+1\right)\left(\mathrm{x}^{2}+\mathrm{x}+1\right)\left(\mathrm{x}^{4}-\mathrm{x}^{2}+1\right) \\
\text { 2. } \mathrm{m}^{24}+\mathrm{m}^{12}+1 & =\left(\mathrm{m}^{12}+\mathrm{m}^{6}+1\right)\left(\mathrm{m}^{12}-\mathrm{m}^{6}+1\right) \\
& =\left(\mathrm{m}^{6}-\mathrm{m}^{3}+1\right)\left(\mathrm{m}^{6}+\mathrm{m}^{3}+1\right)\left(\mathrm{m}^{12}-\mathrm{m}^{6}+1\right) .
\end{aligned}
$$

Lemma 1 is can be extended to Lemma 2 below for a polynomial whose numerical coefficients are greater than 1 .

Lemma 2. $(a x)^{4 n}+(a b x y)^{2 n}+(b y)^{4 n}=\left[(a x)^{2 n}-(a b x y)^{n}+(b y)^{n}\right]\left[(a x)^{2 n}+(a b x y)^{n}+(b y)^{n}\right]$

Example. Find the factors of $4096 x^{12}+(x y)^{6}+y^{12}$.

Solution:

$$
\begin{aligned}
4096 x^{123}+(x y)^{6}+y^{12} & =(2 x)^{12}+(x y)^{6}+y^{12} \\
& =\left[(2 x)^{6}+(x y)^{3}+(y)^{6}\right]\left[(2 x)^{6}-(x y)^{3}+(y)^{6}\right]
\end{aligned}
$$

After illustrating the trinomial conjugates, a polynomial with 5 terms can have a pair of conjugate which is presented as Lemma 3 below. It answers the second problem for finding the factors of $\mathrm{x}^{8 \mathrm{n}}+\mathrm{x}^{6 \mathrm{n}}+\mathrm{x}^{4 \mathrm{n}}+\mathrm{x}^{2 \mathrm{n}}+1$.

Lemma 3. Factors of $x^{8 n}+x^{6 n}+x^{4 n}+x^{2 n}+1$

Let $n$ be a positive integer. Then, $x^{8 n}+x^{6 n}+x^{4 n}+x^{2 n}+1$ is equal to $\left(x^{4 n}+x^{3 n}+\right.$ $\left.\mathrm{x}^{2 \mathrm{n}}+\mathrm{x}^{\mathrm{n}}+1\right)\left(\mathrm{x}^{4 \mathrm{n}}-\mathrm{x}^{3 \mathrm{n}}+\mathrm{x}^{2 \mathrm{n}}-\mathrm{x}^{\mathrm{n}}+1\right)$.

The same proof of the previous lemmas is done here using addition law of equality and factoring by grouping.

Examples. Determine whether each of the following polynomials can be factored or not. If it is, then express it as a product of polynomials.
1. $x^{24}+x^{18}+x^{12}+x^{6}+1$
2. $x^{16}+x^{14}+x^{10}+x^{2}+1$

Solutions:

1. $x^{24}+x^{18}+x^{12}+x^{6}+1=\left(x^{12}+x^{9}+x^{6}+x^{3}+1\right)\left(x^{12}-x^{9}+x^{6}-x^{3}+1\right)$

2. Note that the sequence of the powers of the terms of the polynomial, $\mathrm{x}^{16}+\mathrm{x}^{14}+\mathrm{x}^{10}+\mathrm{x}^{2}+1$, is inconsistent. Thus, Lemma 3 can not be applied. 
Note that the polynomial in Lemma 3 can be extended to the one with numerical coefficients greater than 1 . It is labeled as Lemma 4 as follows.

Lemma 4. Factors of $(a x)^{8 n}+(a x)^{6 n}(b y)^{2 n}+(a x)^{4 n}(b y)^{4 n}+(a x)^{2 n}(b y)^{6 n}+(b y)^{8 n}$

For some real numbers a and $b$, the polynomial $(a x)^{8 n}+(a x)^{6 n}(b y)^{2 n}+(a x)^{4 n}(b y)^{4 n}+$ $(a x)^{2 n}(b y)^{6 n}+(b y)^{8 n}$ is a product of $\left[(a x)^{4 n}+(a x)^{3 n}(b y)+(a x b y)^{2 n}+(a x)^{n}(b y)^{3 n}+(b y)^{4}\right]$ and $\left[(a x)^{4 n}-(a x)^{3 n}(b y)+(a x b y)^{2 n}-(a x)^{n}(b y)^{3 n}+(b y)^{4 n}\right]$.

After studying the sequence of the products of two conjugates with odd number of terms and using the formula of the arithmetic sequence, Theorem 6 provides the view of the form of a product of two conjugates with $\mathrm{m}$ terms. This answers the last problem for finding the factors of $x^{4 n}+(x y)^{4 n-d}+(x y)^{4 n-2 d}+\ldots+(x y)^{d}+y^{4 n}$.

Theorem 5. Factors of $x^{4 n}+(x y)^{4 n-2 d}+(x y)^{4 n-4 d}+\ldots+(x y)^{2 d}+y^{4 n}$

Let $\mathrm{n}$ and $\mathrm{d}$ be any positive integers and $\mathrm{m}$ be any odd integer. Then, the polynomial of $\mathrm{m}$ terms of the form $x^{4 n}+(x y)^{4 n-2 d}+(x y)^{4 n-4 d}+\ldots+(x y)^{2 d}+y^{4 n}$ is factorable into $x^{2 n}+$ $(x y)^{2 n-d}+(x y)^{2 n-2 d}+\ldots+(x y)^{d}+y^{2 n}$ and $x^{2 n}-(x y)^{2 n-d}+(x y)^{2 n-2 d}-\ldots-(x y)^{d}+y^{2 n}$. The numbers of terms of the polynomial, $m$, is equal to $4 n / d+1$.

The same process in proving the previous lemmas is done here.

Examples:

1. Factor $x^{36}+x^{34}+x^{32}+\ldots+x^{18}+\ldots+x^{4}+x^{2}+1$

Solution: Since the degree of the polynomial is $36(n=36)$ which is divisible by 4 and the common difference of the powers of its terms is 2 then using Theorem 5, $\mathrm{m}$ is equal to 19 and $\mathrm{x}^{36}+\mathrm{x}^{34}+\mathrm{x}^{32}+\ldots+\mathrm{x}^{18}+\ldots+\mathrm{x}^{4}+\mathrm{x}^{2}+1$ equals $\left(\mathrm{x}^{18}+\mathrm{x}^{17}+\mathrm{x}^{16}+\ldots+\mathrm{x}+1\right)\left(\mathrm{x}^{18}-\mathrm{x}^{17}+\mathrm{x}^{16}-\ldots-\mathrm{x}+1\right)$.

\section{Summary}

The results in this study illustrate lemmas and theorem of the polynomials of $n$ terms which are product of two conjugates. The first lemma initiated the discoveries of the succeeding lemmas and the theorem in which any polynomial of odd number of terms and whose powers of its terms are in sequence can be product of two conjugates of the same number of terms. Lemma 3 shows that the degree of the polynomial must be divisible by 4 and the powers of its terms must be in sequence. Thus, Theorem 5 is established.

\section{Conclusion and Recommendation}

This study showed another forms of special products whose factors are conjugates. In many algebra textbooks, the introduced conjugates are only binomials. Few knew about the conjugates involving trinomials and radicals. It is in this paper that the other types of conjugates with 3, 5, or even more than 5 terms are shown. This study concluded that the discovery of the product of the other conjugates are essential in search of the other factorable polynomials. Exploring the patterns and sequences of the terms and powers of the polynomials may give discoveries of the concepts involved in it. It is very helpful for enriching the knowledge of anyone and becoming critical thinkers who develop the modern technology that are using today and in the future in finding variety of solutions to several problems and for making our livelihood easier and fast. Thus, this study recommends on encouraging the students to explore more factorable polynomials which are product of conjugates. Furthermore, mathematics teachers, curriculum planners and makers, and other educators should include this study in algebra as an expansion of the types special products. 


\section{Product of conjugates}

\section{References:}

Ababa, Zenaida et., al. (2003). Basic Mathematics Workbook, Third Edition. Marawi City: Mindanao State University - University Book Center.

Coronel, I. C. et. al. (2013). Intermediate Algebra. Philippines: Bookmark.

De Leon, C. M. \& Bernabe, J. G. (2002). Elementary Algebra for First Year. Quezon City: JTW Publishing Co.

De Sagun, O. C. (1999). Algebra 2 with Trigonometry. New Jersey: Prentice Hall Insigne, L. G. et. al. (2003). Intermediate Algebra. Bookman, Inc.

Leithold, Louis. (1992). College Algebra and Trigonometry, (pp. 590, 597 - 604). Canada: Addison - Wesley Publishing Co., Inc.

Swokowski, E. W. \& Mendoza, M. O. (2002). Algebra and Trigonometry with Analytic Geometry, 10th edition. Brooks/Cole.

Vance, E. P. (1975). Modern College Algebra, 3rd edition. Addison-Wesley Publishing Co., Inc.

Copyright (C) 2017 by Normalah P. Solaiman. All rights reserved. No part of this publication may be reproduced, stored in a retrieval system, or transmitted, in any form without the written permission from the author. 